TRANSACTIONS OF THE

AMERICAN MATHEMATICAL SOCIETY

Volume 353, Number 5 , Pages 1959-1970

S 0002-9947(01)02644-7

Article electronically published on January 3, 2001

\title{
SPACES OF RATIONAL LOOPS ON A REAL PROJECTIVE SPACE
}

\author{
JACOB MOSTOVOY
}

\begin{abstract}
We show that the loop spaces on real projective spaces are topologically approximated by the spaces of rational maps $\mathbf{R P}^{1} \rightarrow \mathbf{R} \mathbf{P}^{n}$. As a byproduct of our constructions we obtain an interpretation of the Kronecker characteristic (degree) of an ornament via particle spaces.
\end{abstract}

\section{INTRODUCTION}

It is well-known that rational maps $\mathbf{R} \mathbf{P}^{1} \rightarrow \mathbf{R} \mathbf{P}^{m}$ are dense in the space of all continuous maps from a circle to $\mathbf{R P}^{m}$. Here we will show that the space of all such (basepoint-preserving) rational maps is, in fact, homotopy equivalent to the loop space $\Omega \mathbf{R P}^{m}$. For spaces of maps given by polynomials of bounded degree, we will obtain an estimate of "how well" these spaces approximate $\Omega \mathbf{R P}^{m}$. We consider maps which respect basepoints for convenience only; corresponding statements for basepoint-free rational maps and free loops on $\mathbf{R P}^{m}$ easily follow.

Our results can be viewed as a real version of Segal's theorem [12]. Segal showed that the second loop space $\Omega^{2} \mathbf{C P}^{m}$ on a complex projective space can be topologically approximated by the spaces of basepoint-preserving rational maps from $\mathbf{C P}^{1}$ to $\mathbf{C P}^{m}$. Namely, let $\operatorname{Rat}_{n}(m)$ denote the space of such rational maps of degree $n$ that send the point $\infty \in \mathbf{C P}^{1}$ to some fixed point of $\mathbf{C P}^{m}$ with the topology of a subset of $\Omega^{2} \mathbf{C} \mathbf{P}^{m}$. It is convenient to choose $(1,1, \ldots, 1)$ as a basepoint in $\mathbf{C P}^{m}$; then every basepoint-preserving rational map can be given by a collection of monic polynomials of the same degree. Let $\left(\Omega^{2} \mathbf{C P}^{m}\right)_{n}$ be the component of $\Omega^{2} \mathbf{C P}^{m}$ which parametrizes maps of degree $n$. Then the following is true:

Theorem 1.1 ([12]). The natural inclusion

$$
\operatorname{Rat}_{n}(m) \hookrightarrow\left(\Omega^{2} \mathbf{C P}^{m}\right)_{n}
$$

is a homotopy equivalence up to dimension $n(2 m-1)$.

This result was later generalized to spaces of maps into a wide class of complex manifolds, see [1], 4], 2].

Segal has also proved a real version of this theorem, which is different from ours. Denote by $\operatorname{RRat}_{n}(m)$ the subspace of $\operatorname{Rat}_{n}(m)$ of maps which commute with complex conjugation. Let $\operatorname{RRat}_{n, k}(1)$ be the component of $\operatorname{RRat}_{n}(1)$ which

Received by the editors June 2, 1998 and, in revised form, October 18, 1999.

2000 Mathematics Subject Classification. Primary 26C15, 55P35.

Key words and phrases. Loop space, rational map, ornament, Kronecker characteristic.

(C)2001 American Mathematical Society 
parametrizes maps whose restriction to the real line has degree $k$. Also, let $\Omega^{2}\left(\mathbf{C P}^{m}, \mathbf{R P}^{m}\right)$ be the space of continuous basepoint-preserving maps

$$
\left(D^{2}, S^{1}, *\right) \rightarrow\left(\mathbf{C P}^{m}, \mathbf{R} \mathbf{P}^{m}, *\right) .
$$

Such maps can be thought of as continuous conjugation-equivariant maps

$$
\mathbf{C P}^{1} \rightarrow \mathbf{C P}^{m} \text {. }
$$

Finally, let $\Omega^{2}\left(\mathbf{C P}^{1}, \mathbf{R} \mathbf{P}^{1}\right)_{n, k}$ be the connected component of $\Omega^{2}\left(\mathbf{C P}^{1}, \mathbf{R} \mathbf{P}^{1}\right)$ which parametrizes maps that have degree $n$ as maps $\mathbf{C} \mathbf{P}^{1} \rightarrow \mathbf{C P}^{1}$ and degree $k$ when restricted to the real axis.

Theorem 1.2 ([12]). The natural inclusion

$$
\operatorname{RRat}_{n, k}(1) \hookrightarrow \Omega^{2}\left(\mathbf{C P}^{1}, \mathbf{R P}^{1}\right)_{n, k}
$$

is a homotopy equivalence up to dimension $\frac{1}{2}(n-|k|)$.

Now we will describe our real analogue of Theorem 1.1 .

The restriction of any map $f \in \operatorname{RRat}_{n}(m)$ to the real axis defines a map

$$
f_{\mathbf{R}}: \mathbf{R} \mathbf{P}^{1} \rightarrow \mathbf{R P}^{m} \text {. }
$$

In fact, $f_{\mathbf{R}}$ uniquely determines $f$, so there is an $\operatorname{inclusion}_{\operatorname{RRat}_{n}}(m) \hookrightarrow \Omega \mathbf{R} \mathbf{P}^{m}$. The image of this inclusion is not closed in $\Omega \mathbf{R} \mathbf{P}^{m}$. For example, the sequence of maps $g_{\epsilon}=\frac{x^{2}+1+\epsilon}{x^{2}+1}$ does not have a limit in $\operatorname{RRat}_{2}(1)$ as $\epsilon$ tends to 0 . (Here $x$ is the coordinate on the affine piece of $\mathbf{R} \mathbf{P}^{1}$.) However, inside $\Omega \mathbf{R P}^{1}$ it converges to a constant map.

Definition 1.3. The space $\operatorname{rat}_{n}(m)$ of real rational maps from $\mathbf{R} \mathbf{P}^{1}$ to $\mathbf{R} \mathbf{P}^{m}$ is the closure of $\operatorname{RRat}_{n}(m)$ in $\Omega \mathbf{R P} \mathbf{P}^{m}$.

From what follows it will become clear that all points of $\operatorname{rat}_{n}(m)$ correspond to rational maps given by polynomials of degrees $k \leqslant n$, where $k$ and $n$ have the same parity.

First we will look at the case $m=1$.

Theorem 1.4. The space rat $_{n}(1)$ consists of $n+1$ contractible components, indexed by the topological degree of the map $\mathbf{R P}^{1} \rightarrow \mathbf{R} \mathbf{P}^{1}$, which ranges from $-n$ to $n$ and has the same parity as $n$.

Remark 1.5. The components of $\Omega \mathbf{R} \mathbf{P}^{1}$ are contractible.

When $m>1$ the space $\operatorname{rat}_{n}(m)$ is connected. Denote by $\left(\Omega \mathbf{R P}^{m}\right)_{k}$, where

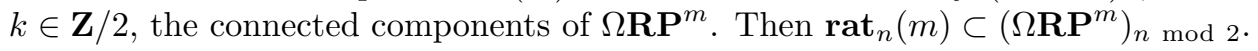

Theorem 1.6. For $m>1$ the natural inclusion

$$
\operatorname{rat}_{n}(m) \hookrightarrow\left(\Omega \mathbf{R P}^{m}\right)_{n \bmod 2}
$$

is a homotopy equivalence up to dimension $n(m-1)$.

The proof goes along the lines of Segal's proof of Theorem 1.1 In section 2 we define two types of configuration spaces which describe divisors associated to real rational maps; we also define the stabilized spaces $\operatorname{rat}_{\infty}(m)$. Section 3 deals with maps from $\mathbf{R P}^{1}$ to $\mathbf{R P}^{1}$. In section 4 we describe the stabilization results which prove Theorem 1.6 for $m>2$. In section 5 we consider the action of $\pi_{1} \mathbf{r a t}_{n}(2)$ on the higher homotopy groups and finish the proof of Theorem 1.6. Section 6 
has no direct relevance to rational maps and is a byproduct of the constructions in section 5: we define an integer-valued invariant of ornaments (see [13], [15]) which is interpreted as the degree of a certain map $S^{2} \rightarrow S^{2}$.

Our methods and results are closely related to recent work on the complements of discriminants. Apart from those cited above, we should mention the results of Vassiliev [14, 15] and the work of Guest, Kozlowski and Yamaguchi [5]. It was pointed out by the referee that when $m \geqslant 3$ our Proposition 4.1 is a particular case of Theorem 3.8 in [5. We should also say that our main theorem follows from the results recently obtained in [8], [16] by Kozlowski and Yamaguchi. Our technique, however, seems to be sufficiently different as to be of independent interest.

\section{Configurations of Real Divisors}

Maps in $\operatorname{RRat}_{n}(m)$ can be parametrized by collections of $m+1$ positive real divisors of degree $n$ in $\mathbf{C}$ subject to the following condition: the intersection of the supports of all $m+1$ divisors is empty. (A positive real divisor in $\mathbf{C}$ is a divisor of all the roots, both real and complex, of a real polynomial.)

If we wish to find a configuration space which would parametrize maps in $\operatorname{rat}_{n}(m)$, we must substitute this condition for a weaker one, namely: the intersection of supports of all $m+1$ divisors must be disjoint from the real axis. We denote the space of such collections of divisors by $D(n, m)$.

Choose $z \in \mathbf{C}, \operatorname{Im}(z) \neq 0$. There is an inclusion map

$$
D(n, m) \stackrel{i_{n}^{\prime}}{\longrightarrow} D(n+2, m)
$$

defined by adding the divisor $z+\bar{z}$ to each of the $m+1$ divisors.

Notice that we do not obtain a 1-1 correspondence between $\operatorname{rat}_{n}(m)$ and $D(n, m)$; for example, the pairs of polynomials $\left(x^{2}+1, x^{2}+1\right)$ and $\left(x^{2}+2, x^{2}+2\right)$ define the same (constant) map in $\operatorname{rat}_{2}(1)$. However, we have a continuous map

$$
P: D(n, m) \rightarrow \operatorname{rat}_{n}(m) .
$$

Roughly speaking, if we think of points of $D(n, m)$ as of collections of particles of several kinds, we allow particles of different kinds to cancel out provided they are not on the real axis. The effect of this cancellation is that the degree of all divisors drops by 2 , so we have a commutative diagram

$$
\begin{array}{ccc}
D(n, m) & \stackrel{i_{n}^{\prime}}{\longrightarrow} & D(n+2, m) \\
\downarrow P & & \downarrow P \\
\operatorname{rat}_{n}(m) & \stackrel{i_{n}}{\longrightarrow} & \operatorname{rat}_{n+2}(m)
\end{array}
$$

Notice that the inclusion map $i_{n}$ is canonical. Clearly,

$$
\operatorname{rat}_{n}(m) \backslash \operatorname{rat}_{n-2}(m)=\operatorname{RRat}_{n}(m) .
$$

There is another space of real divisors which will be of interest for us. Let $\mathbf{R}^{(n)}$ denote the $n$-fold symmetric product of $\mathbf{R}$. We write points in $\mathbf{R}^{(n)}$, i.e. divisors on $\mathbf{R}$, as linear combinations $\sum k_{i} x_{i}$ of points $x_{i} \in \mathbf{R}$ with non-negative (but possibly zero) multiplicities. (Here, obviously, $\sum k_{i}=n$.) Define the subset $S(n, m)$ of the $(m+1)$-fold Cartesian product $\left[\mathbf{R}^{(n)}\right]^{m+1}$ by the condition that the intersection of supports of all $m+1$ divisors is empty. We define the configuration space $T(n, m)$ 
of mod 2 particles on the real line as the quotient of $S(n, m)$ by the following equivalence relation:

$$
\left(\sum_{i} k_{i}^{0} x_{i}^{0}, \ldots, \sum_{i} k_{i}^{m} x_{i}^{m}\right) \sim\left(\sum_{i} l_{i}^{0} x_{i}^{0}, \ldots, \sum_{i} l_{i}^{m} x_{i}^{m}\right)
$$

if $k_{q}^{p} \equiv l_{q}^{p}(\bmod 2)$ for all $p, q$.

The points of $T(n, m)$ are, essentially, collections of particles of $m+1$ different kinds on $\mathbf{R}$, such that two particles of the same kind annihilate each other and such that $m+1$ particles of different kinds cannot collide in the same point.

Define a map

$$
D(n, m) \rightarrow T(n, m)
$$

by sending a collection of polynomials which is defined by a point in $D(n, m)$ to the collection of their real root systems with multiplicities reduced modulo 2 . It is easy to check that this map is continuous and factors through $\operatorname{rat}_{n}(m)$. There is a commutative diagram

$$
\begin{array}{ccc}
\operatorname{rat}_{n}(m) & \stackrel{i_{n}}{\hookrightarrow} & \operatorname{rat}_{n+2}(m) \\
\downarrow Q & & \downarrow Q \\
T(n, m) & \stackrel{i_{n}^{\prime \prime}}{\hookrightarrow} & T(n+2, m)
\end{array}
$$

Both inclusions in this diagram are canonical.

There is another kind of inclusion maps

$$
j_{n}^{\prime}: D(n, m) \hookrightarrow D(n+1, m),
$$

defined by "adding real points at infinity". We can think of $D(n, m)$ as consisting of collections of divisors that are contained in an open disk of radius $n$ with the center at 0 . Choose $x_{i} \in \mathbf{R}, 0 \leqslant i \leqslant m$, such that $n<x_{0}<x_{1}<\ldots<x_{m}<n+1$. Then the inclusion $j_{n}^{\prime}$ is defined by adding $x_{i}$ to the $i$-th divisor in the collection for each $i$.

There are induced inclusions

$$
j_{n}: \operatorname{rat}_{n}(m) \hookrightarrow \operatorname{rat}_{n+1}(m)
$$

and

$$
j_{n}^{\prime \prime}: T(n, m) \hookrightarrow T(n+1, m) .
$$

None of these maps are canonical; it is easy to see, however, that, for $m>1, j_{n+1}^{\prime} j_{n}^{\prime}$ is homotopic to $i_{n}^{\prime}$, and similarly for $j_{n}$ and $j_{n}^{\prime \prime}$.

Now for $m>1$ we can define the space $\operatorname{rat}_{\text {odd }}(m)$ as the direct limit of the sequence

$$
\operatorname{rat}_{1}(m) \stackrel{i_{1}}{\hookrightarrow} \operatorname{rat}_{3}(m) \stackrel{i_{3}}{\hookrightarrow} \ldots
$$

The spaces $\operatorname{rat}_{\mathrm{ev}}(m), D$ (odd, $\left.m\right), D(\mathrm{ev}, m), T(\mathrm{odd}, m)$ and $T(\mathrm{ev}, m)$ are defined similarly as direct limits. By $\mathbf{r a t}_{\infty}(m)$ and $T(\infty, m)$ we denote the disjoint unions $\operatorname{rat}_{\text {odd }}(m) \cup \operatorname{rat}_{\mathrm{ev}}(m)$ and $T(\mathrm{odd}, m) \cup T(\mathrm{ev}, m)$ respectively. As before, the collections of divisors in $D(\mathrm{ev}, m)$ can be thought of as lying in an open disk of radius 1 with the center at 0 . Adding a point $x_{k}=2+k$ to the $k$ th divisor for all $0 \leqslant k \leqslant m$, we get a map $j_{\mathrm{ev}}^{\prime}$ from $D(\mathrm{ev}, m)$ to $D$ (odd, $m$ ), which is clearly a homotopy equivalence. This map also descends to homotopy equivalences

$$
j_{\mathrm{ev}}: \operatorname{rat}_{\mathrm{ev}}(m) \rightarrow \operatorname{rat}_{\text {odd }}(m)
$$


and

$$
\left.j_{\mathrm{ev}}^{\prime \prime}: T(\mathrm{ev}, m) \rightarrow T \text { (odd, } m\right) .
$$

The spaces $D(n, m), \operatorname{rat}_{n}(m)$, and $T(n, m)$ have homotopy types of CWcomplexes. This is clear for $D(n, m)$ as it is a smooth manifold; for $\operatorname{rat}_{n}(m)$ and $T(n, m)$ it can be established by an argument similar to the proof of Lemma 2.2 of [10.

The reason for considering the configuration spaces $D(n, m)$ and $T(n, m)$ is the following

Proposition 2.1. The maps

$$
D(n, m) \stackrel{P}{\longrightarrow} \operatorname{rat}_{n}(m) \stackrel{Q}{\longrightarrow} T(n, m)
$$

are homotopy equivalences for all $n$ and $m$, including $n=\infty$.

Proof. Essentially, the reason $P$ and $Q$ are homotopy equivalences is that they have contractible fibers. As we have seen above, the space $\operatorname{rat}_{n}(m)$ has a canonical filtration by subspaces $\operatorname{rat}_{k}(m)$, where $k \leqslant n$ and $k \equiv n(\bmod 2)$. The fiber of $P$ over $\boldsymbol{r a t}_{k}(m) \backslash \mathbf{r a t}_{k-2}(m)=\operatorname{RRat}_{k}(m)$ is the $\frac{1}{2}(n-k)$-fold symmetric product of the open upper half-plane, i.e. $\mathbf{R}^{n-k}$. In fact, $P^{-1}\left(\operatorname{RRat}_{k}(m)\right)$ is the direct product $\operatorname{RRat}_{k}(m) \times \mathbf{R}^{n-k}$. The space $D(n, m)$ can be triangulated in such a way that the inverse image $P^{-1}\left(\boldsymbol{r a t}_{k}(m)\right)$ is a subcomplex, so the inclusion $P^{-1}\left(\operatorname{rat}_{k}(m)\right) \hookrightarrow$ $D(n, m)$ is a cofibration. Let $p_{k}$ be the sequence of maps which collapse the fibers of $P$ over $\operatorname{RRat}_{k}(m)$ consecutively. It is a sequence of homotopy equivalences. On the other hand, $P=p_{n} p_{n-2} \ldots$

The argument for $T(n, m)$ is similar. The space $T(n, m)$ is graded by subspaces $T_{\mathbf{k}}(n, m)$, where $\mathbf{k}=\left(k_{0}, \ldots, k_{m}\right)$ is a set of integers such that $0 \leqslant k_{i} \leqslant n$ and $k \equiv n(\bmod 2)$. A point of $T(n, m)$ belongs to $T_{\mathbf{k}}(n, m)$ if it has exactly $k_{i}$ particles of the $i$ th kind.

The closure of $T_{\mathbf{k}}(n, m)$ is contained in the union $\bigcup_{\mathbf{l} \leqslant \mathbf{k}} T_{\mathbf{l}}(n, m)$; here by $\mathbf{l} \leqslant \mathbf{k}$ we mean $l_{i} \leqslant k_{i}$ for any $i$. The restriction of $Q P$ to $P^{-1} Q^{-1}\left(T_{\mathbf{k}}(n, m)\right)$ is clearly a homotopy equivalence and, for the same reason as before, the inclusion

$$
P^{-1} Q^{-1}\left(\bigcup_{\mathbf{l} \leqslant \mathbf{k}} T_{\mathbf{l}}(n, m)\right) \hookrightarrow D(n, m)
$$

is a cofibration. Collapsing the fibers of $Q P$ consecutively, we get a chain of homotopy equivalences, so $Q P$ (and, hence, $Q$ ) is itself a homotopy equivalence.

As an immediate corollary we get that for $m>1$ the spaces $\operatorname{rat}_{n}(m)$ are connected, and for $m>2$ they are simply-connected. Indeed, $D(n, m)$ is obtained by removing a triangulable subset of codimension $m$ from $\mathbf{R}^{n(m+1)}$.

\section{SPACES OF MAPS $\mathbf{R P}^{1} \rightarrow \mathbf{R P}^{1}$}

It is well-known that $\operatorname{RRat}_{n}(1)$ has $n+1$ connected components. They are indexed by the degree of maps $\mathbf{R} \mathbf{P}^{1} \rightarrow \mathbf{R} \mathbf{P}^{1}$, which ranges from $-n$ to $n$ and has the same parity as $n$, see [3] and [12]. The same is true for $\operatorname{rat}_{n}(1)$, as $\operatorname{RRat}_{n}(1) \subset$ $\operatorname{rat}_{n}(1)$ is a dense subset. Alternatively, it is easy to see directly that $T(n, 1)$ has exactly $n+1$ components.

Theorem 1.4 follows from

Proposition 3.1. Connected components of $T(n, 1)$ are contractible. 
Proof. Points of $T(n, 1)$ are pairs of disjoint mod 2 divisors on a real line. We can treat them as sets of particles of different "charges", such that particles of the same charge annihilate each other and particles of different charges cannot collide. Let us extend the analogy.

At the time $t=0$ let all the particles be at rest. We introduce three forces: gravity with the potential $x^{2}$, friction which is proportional to the velocity of the particle, and interaction between particles. A particle interacts only with its immediate neighbors; it attracts particles of the same charge and repels particles of the opposite charge; the magnitude of this force is $\frac{1}{r}$, where $r$ is the distance between particles. The dynamics of this system defines a flow on $T(n, 1)$. As particles of the same charge annihilate each other, the interaction forces change discontinuously, but, as the force is essentially the second derivative of the coordinate, the flow is continuous, at least on any compact subset. Calculation shows that there is exactly one state of equilibrium for each connected component of $T(n, 1)$, so as $t \rightarrow \infty$ we get a homotopy of any map $S^{k} \rightarrow T(n, 1)$ to a constant map. Hence, all components of $T(n, 1)$ have trivial homotopy groups, and this implies that they are contractible.

\section{The Homotopy EQUivalence $\operatorname{rat}_{\infty}(m) \hookrightarrow \Omega \mathbf{R} \mathbf{P}^{m}$ AND HOMOLOGY STABILIZATION}

Proposition 4.1. For all $m$ the natural inclusion

$$
\operatorname{rat}_{\infty}(m) \hookrightarrow \Omega \mathbf{R} \mathbf{P}^{m}
$$

is a homotopy equivalence.

Proof. The statement of the proposition for $m=1$ follows from Theorem 1.4 so we will assume that $m \geqslant 2$.

Let $y_{0}, \ldots, y_{m}$ be the homogeneous coordinates in $\mathbf{R P}^{m}$. Denote by $\Sigma$ the subspace of loops which have an infinite number of intersection points with some of the hyperplanes $y_{k}=0$. The inclusion map $\Omega \mathbf{R} \mathbf{P}^{m} \backslash \Sigma \hookrightarrow \Omega \mathbf{R} \mathbf{P}^{m}$ is a homotopy equivalence. The reason for this is that $\Omega \mathbf{R} \mathbf{P}^{m}$ can be thought of as an infinitedimensional manifold and $\Sigma$ is a subset of infinite codimension, so removing $\Sigma$ does not change the homotopy type of $\Omega \mathbf{R} \mathbf{P}^{m}$. However, rather than making this argument precise, we will sketch a direct proof.

Every loop on $\mathbf{R P}^{m}$ can be lifted to a loop or a path on $S^{m}=\left\{y_{0}, \ldots, y_{m} \mid\right.$ $\left.y_{0}^{2}+\ldots+y_{m}^{2}=1\right\}$. First, with the help of some self-homeomorphism of $S^{m}$ we deform the space $\Omega \mathbf{R} \mathbf{P}^{m} \backslash \Sigma$ into the space of loops (paths) on $S^{m}$ that have not more than a finite number of intersections with any of the sets $y_{k}=\epsilon$ for some small $\epsilon$. Notice that any piecewise geodesic (in the standard metric) path on $S^{m}$ has only a finite number of intersections with $y_{k}=\epsilon$ for any $k$. Now one can use piecewise geodesic approximations, and the methods of [11] can be applied. The argument is identical to the proof of Theorem 17.1 of [11, with the space of piecewise smooth loops replaced by $\Omega \mathbf{R} \mathbf{P}^{m} \backslash \Sigma$.

Define a map $q: \Omega \mathbf{R P}^{m} \backslash \Sigma \stackrel{q}{\longrightarrow} T(\infty, m)$ by sending a loop $\omega$ to the collection of mod 2 divisors $\left(\zeta_{0}, \ldots, \zeta_{m}\right)$, where $\zeta_{k}$ consists of the values of the parameter $x$ at which $\omega$ intersects the hyperplane $y_{k}=0$; the coefficient of $x$ in $\zeta_{k}$ is 1 if $\omega$ crosses $y_{k}=0$ near $x$ and 0 otherwise. 
In the commutative diagram

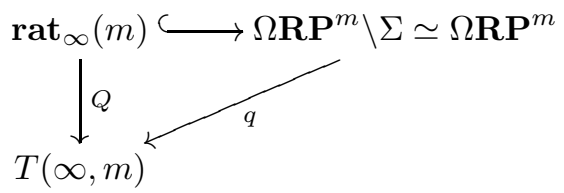

$Q$ is a homotopy equivalence, so all maps $\pi_{*} \mathbf{r a t}_{\infty}(m) \hookrightarrow \pi_{*} \Omega \mathbf{R} \mathbf{P}^{m}$ are injective.

On the other hand, the Weierstraß approximation theorem implies that the above maps are surjective. Indeed, consider the inclusion of the even components rat $_{\mathrm{ev}}(m) \hookrightarrow\left(\Omega \mathbf{R P}^{m}\right)_{0}=\Omega S^{m}$. Each loop on $S^{m}$ can be represented by $m+1$ functions $f_{0}(x), \ldots, f_{m}(x)$ such that $\lim _{x \rightarrow \infty} f_{i}(x)=1$ for any $i$ and $\sum_{0}^{m} f_{i}(x)^{2}=m+1$. If we identify $\mathbf{R} \cup\{\infty\}$ with $S^{1}$ by substituting $x=\tan \frac{\alpha}{2}$, the functions $f_{i}$ become continuous functions on $S^{1}$ and, hence, can be uniformly approximated by trigonometric polynomials. It is easy to check that trigonometric polynomials in $\alpha$ are rational functions in $x$, so any loop on $S^{m}$ can be uniformly approximated by rational loops. The same argument establishes that any map $S^{k} \rightarrow\left(\Omega \mathbf{R P}^{m}\right)_{0}$ can be uniformly approximated by maps $S^{k} \rightarrow \mathbf{r a t}_{\mathrm{ev}}(m)$. This is enough to show that every element of $\pi_{k}\left(\Omega \mathbf{R} \mathbf{P}^{m}\right)_{0}$ is an image of some element of $\pi_{k} \mathbf{r a t}_{\mathrm{ev}}(m)$. The same is true for the odd components, as there is a homotopy commutative diagram

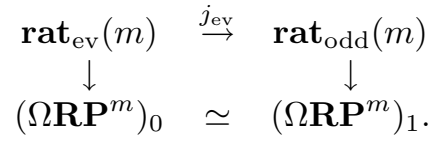

Proposition 4.2. The inclusion $\operatorname{map} j_{n}: \operatorname{rat}_{n}(m) \hookrightarrow \operatorname{rat}_{n+1}(m)$ is a homology equivalence up to dimension $n(m-1)$ for all $m>1$ and all $n>0$.

As $j_{n+1} j_{n}$ is homotopic to the inclusion $i_{n}: \operatorname{rat}_{n}(m) \hookrightarrow \operatorname{rat}_{n+2}(m)$, it is enough to prove that $i_{n}$ is a homology equivalence up to dimension $n(m-1)$. In view of Proposition 2.1] this follows from the corresponding statement for the map $i_{n}^{\prime}: D(n, m) \hookrightarrow D(n+2, m)$. The proof of the latter repeats Segal's proof of Proposition 5.1 in [12] almost word-for-word; we refer to [12] for details.

In the case when $m>2, \operatorname{rat}_{n}(m)$ is simply-connected and so $j_{n}$ is a homotopy equivalence up to dimension $n(m-1)$. In order to show that this is also the case for $m=2$ it is necessary to check that $\operatorname{rat}_{n}(2)$ is simple up to dimension $n$, i.e. that for $k<n$ the action of $\pi_{1} \boldsymbol{r a t}_{n}(2)$ on $\pi_{k} \mathbf{r a t}_{n}(2)$ is trivial.

\section{SPACES OF MAPS $\mathbf{R P}^{1} \rightarrow \mathbf{R P}^{2}$}

First let us consider the spaces $\operatorname{rat}_{1}(2)$ and $\operatorname{rat}_{2}(2)$. The space $D(1,2)$ is $\mathbf{R}^{3}$ with the diagonal line removed, so $\operatorname{rat}_{1}(2) \simeq S^{1}$. A generator of $\pi_{1} D(1,2)$ can be represented by a loop

$$
t \rightarrow(x+\cos t, x+\sin t, x) ; t \in[0,2 \pi] .
$$

The space $\operatorname{rat}_{2}(2)$ is homotopy equivalent to a torus with one of the meridians collapsed; so, in particular, $\pi_{1} \mathbf{r a t}_{2}(2)=\mathbf{Z}$. This can be seen as follows.

Points in $D(2,2)$ can be treated as maps $\mathbf{R} \rightarrow \mathbf{R}^{3}$. Images of these maps are parabolas (possibly degenerated to rays) which miss 0 and whose asymptotic direction is $(1,1,1)$. Let $y_{1}, y_{2}, y_{3}$ be the coordinates in $\mathbf{R}^{3}$. The map from $D(2,2)$ 
to $\left(\mathbf{R}^{2} \backslash 0\right) \times\left(\mathbf{R}^{2} \backslash 0\right)$ with the diagonal collapsed is given by sending the first point of intersection of the parabola with the plane $y_{1}+y_{2}+y_{3}=0$ to the first copy of $\mathbf{R}^{2} \backslash 0$, and the second point of intersection to the second copy. If the parabola does not intersect the plane at all, we map it into the collapsed diagonal. This map is a quasifibration with contractible fibres, and hence a homotopy equivalence.

A generator of $\pi_{1} D(2,2)$ can be represented by a loop

$$
t \rightarrow\left(x^{2}+x+\cos t+\frac{1}{2} \sin t, x^{2}+x+\cos t-\frac{1}{2} \sin t, x^{2}-x+\cos t\right),
$$

where $t \in[0,2 \pi]$.

Proposition 5.1. $\pi_{1} \boldsymbol{r a t}_{n}(2)=\mathbf{Z}$ for any $n>0$.

Proof. The loops of even degree in $\mathbf{R P}^{2}$ can be lifted to loops on a 2-sphere; (we think of the basepoint as the north pole); loops of odd degree can be lifted to paths from the north pole to the south pole. Fix a group structure on the equator $S^{1}$. We define a map $\operatorname{rat}_{n}(2) \rightarrow S^{1}$ by sending a rational map $f$ to the alternating sum $b_{1}-b_{2}+b_{3}-\ldots$, where $b_{i}$ is the $i$-th point of intersection of the image of $f$ with the equator. (Here we count the intersection points with multiplicities so, for example, a double point of intersection will have no effect on the sum.) It can be verified directly that the composite maps

$$
\pi_{1} \operatorname{rat}_{1}(2) \stackrel{I_{2 k+1}}{\longrightarrow} \pi_{1} \operatorname{rat}_{2 k+1}(2) \rightarrow \pi_{1} S^{1}
$$

and

$$
\pi_{1} \operatorname{rat}_{2}(2) \stackrel{I_{2 k}}{\longrightarrow} \pi_{1} \operatorname{rat}_{2 k}(2) \rightarrow \pi_{1} S^{1}
$$

are isomorphisms. Hence the maps $I_{2 k+1}: \pi_{1} \boldsymbol{r a t}_{1}(2) \rightarrow \pi_{1} \boldsymbol{r a t}_{2 k+1}(2)$ and $I_{2 k}$ : $\pi_{1} \operatorname{rat}_{2}(2) \rightarrow \pi_{1} \operatorname{rat}_{2 k}(2)$ are injective.

Notice that the equator considered as a group acts freely on the spaces $\operatorname{rat}_{2 k+1}(2)$ by rotating the sphere. This action commutes with the map $\operatorname{rat}_{2 k+1}(2) \rightarrow S^{1}$ defined above; moreover, the orbits map onto $S^{1}$ homeomorphically, so $\operatorname{rat}_{2 k+1}(2)$ splits as a product of a circle with the quotient space by the action. This will imply that the spaces $\boldsymbol{r a t}_{2 k+1}(2)$ are homotopy simple in all dimensions as soon as we have proved that $\pi_{1} \mathbf{r a t}_{2 k+1}(2)=\mathbf{Z}$. This argument fails for the loops of even degree; and, indeed, one can show that $\pi_{1} \mathbf{r a t}_{2}(2)$ acts non-trivially on $\pi_{2} \mathbf{r a t}_{2}(2)$.

Remark 5.2. It is well-known that there is a decomposition

$$
\Omega S^{2} \simeq S^{1} \times \Omega S^{3}
$$
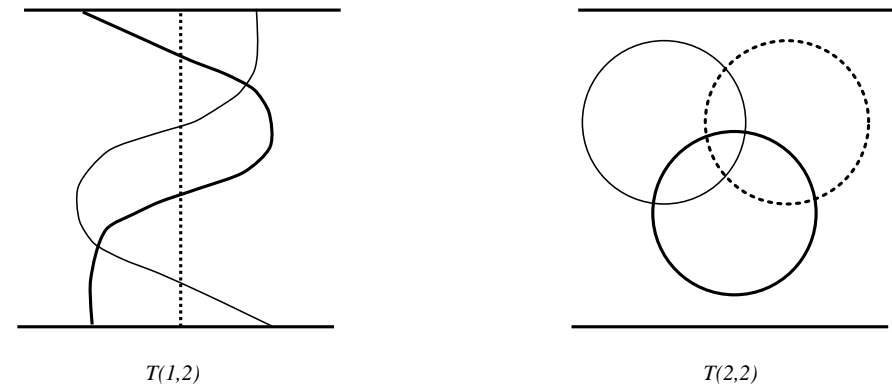

Figure 1. Generators of $\pi_{1} T(1,2)$ and $\pi_{1} T(2,2)$. 
associated to the the Hopf fibration. Yamaguchi recently proved in 16] that $\mathbf{r a t}_{2 k+1}(2)$ is homotopy equivalent to the product of $S^{1}$ with the $k$ th stage of the James filtration on $\Omega S^{3}$.

Now, rather than looking at loops on $\operatorname{rat}_{n}(2)$ we will consider loops on $T(n, 2)$. They can be presented in a braid-like fashion; Figure 1 shows generators of $\pi_{1} T(1,2)$ and $\pi_{1} T(2,2)$. A loop is obtained by moving the horizontal line down and registering the intersection with the "braid". Strictly speaking, the intersections must be taken with mod 2 multiplicities. For example, on the right-hand diagram of Figure 1 the multiplicity of the intersection with the horizontal lines at the "top" and "bottom" points of each circle is 0 .

To prove that the maps $I_{n}$ are surjective we show directly that any loop in $\boldsymbol{r a t}_{2 k+1}(2)$ or $\boldsymbol{r a t}_{2 k}(2)$ can be deformed into $\boldsymbol{r a t}_{1}(2)$ or $\boldsymbol{r a t}_{2}(2)$ respectively.

Let $\zeta(t)=\left(\zeta_{1}(t), \zeta_{2}(t), \zeta_{3}(t)\right)$ be a loop on $T(2 k+1,2)$. By "support of a family of $\bmod 2$ divisors $\zeta_{i}(t)$ ", $i=1,2,3$, we will mean the closure of the set $\operatorname{supp}\left(\zeta_{i}(t)\right)$ in $\mathbf{R} \times[0,1]$. Without loss of generality assume that supports of families of divisors $\zeta_{2}(t)$ and $\zeta_{3}(t)$ intersect in a finite number of points $a_{i}$, corresponding to distinct values of parameter $t_{i}$, where $0 \leqslant i \leqslant C$ for some integer $C$.

First of all we deform $\zeta(t)$ in such a way that $\operatorname{supp}\left(\zeta_{1}(t)\right)$ for each fixed $t$ consists of one point. This is done in two steps.

First we find such an $\epsilon$ that:

- the support of the family $\zeta_{1}(t)$ is disjoint from the rectangle

$$
R=\left[a_{i}-\epsilon, a_{i}+\epsilon\right] \times\left[t_{i}-2 \epsilon, t_{i}+2 \epsilon\right]
$$

in $\mathbf{R} \times[0,1]$ for any $0 \leqslant i \leqslant C$;

- $t_{i+1}-t_{i}>4 \epsilon$ for $0 \leqslant i<C$ and $t_{0}>2 \epsilon$ and $t_{C}<1-2 \epsilon$.

Let us fix $i$ for the moment. On the interval of the parameter $\left|t-t_{i}\right|<2 \epsilon$ the divisor $\zeta_{1}(t)$ can be expressed as a sum $\zeta_{1}^{\prime}(t)+\zeta_{1}^{\prime \prime}(t)$ such that $\zeta_{1}^{\prime}(t)$ lies to the left and $\zeta_{1}^{\prime \prime}(t)$ lies to the right of $a_{i}$. As the mod 2 degree of the divisor $\zeta_{1}(t)$ is 1 for any $t$, either $\zeta_{1}^{\prime}(t)$ or $\zeta_{1}^{\prime \prime}(t)$ has mod 2 degree 0 for any $t \in\left[t_{i}-2 \epsilon, t_{i}+2 \epsilon\right]$. Let, for example, the mod 2 degree of $\zeta_{1}^{\prime \prime}(t)$ be equal to 0 .

Let $N$ be so big that the support of the family $\zeta_{1}(t)$ lies in $(-\infty, N)$. Define a homotopy $\phi_{u}$ from $\mathbf{R} \times[0,1] \backslash R$ to itself such that:

- it is constant everywhere apart from the rectangle $\left[a_{i}+\epsilon, N\right] \times\left[t_{i}-2 \epsilon, t_{i}+2 \epsilon\right]$;

- it carries the rectangle $\left[a_{i}+\epsilon, N\right] \times\left[t_{i}-\epsilon, t_{i}+\epsilon\right]$ onto the interval $N \times\left[t_{i}-\epsilon, t_{i}+\epsilon\right]$; see Figure 2.

We change $\zeta(t)$ by deforming $\zeta_{1}(t)$ with the help of the homotopy $\phi_{u}$ and leaving $\zeta_{2}(t)$ and $\zeta_{3}(t)$ fixed. The effect of this operation is that the points of $\zeta_{1}(t)$ to the right of $a_{i}$ cancel out.

After performing this deformation for each $i$ we do the second step. Let $g(t)$, $t \in[0,1]$, be a function such that:

- for each $i$ we have $g\left(t_{i}\right)<a_{i}$ if the $\bmod 2$ degree of the divisors $\zeta_{1}^{\prime}(t)$ is 1 , and $g\left(t_{i}\right)>a_{i}$ otherwise;

- the graph of $g(t)$ intersects the support of the family of divisors $\zeta_{3}\left(t_{i}\right)$ in a finite number of points.

Now, let $\psi_{u}$ be a retraction of $\mathbf{R} \times[0,1]$ onto the graph of $g(t)$ which preserves lines $t=$ const. We deform $\zeta(t)$ by pushing $\zeta_{1}(t)$ towards the curve $g(t)$ with the help of $\psi_{u}$. 

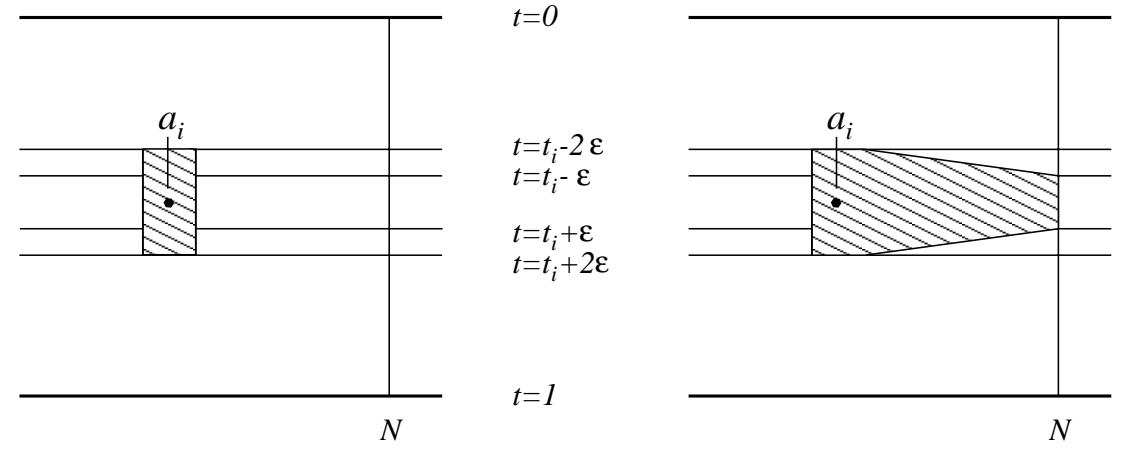

Figure 2. The homotopy $\phi_{u}$ at $u=0$ and $u=1$.

The result of the above tricks is that for any $t$ the deformed $\zeta_{1}(t)$ consists of one point only, and $\zeta_{2}(t)$ and $\zeta_{3}(t)$ don't change. So after performing similar operations with $\zeta_{2}(t)$ and $\zeta_{3}(t)$ we get a loop in $T(1,2)$ which is homotopic to $\zeta(t)$.

The proof that any loop in $T(2 k, 2)$ can be deformed into $T(2,2)$ is similar, but easier; we leave it to the reader.

We have already seen that the spaces $\operatorname{rat}_{n}(2)$ are homotopy simple for $n$ odd. The proof of Theorem [1.6 will be finished as soon as we have proved

Proposition 5.3. For $n$ even the space $\boldsymbol{r a t}_{n}(2)$ is homotopy simple up to dimension $n$.

Proof. By Proposition $5.1 \pi_{1} \boldsymbol{r a t}_{n}(2)=\mathbf{Z}$, so $\boldsymbol{r a t}_{n}(2)$ is homotopy simple up to dimension 1.

Suppose we know that for some $k<n$ the $\operatorname{space} \boldsymbol{r a t}_{n}(2)$ is homotopy simple up to dimension $k$. By Proposition4.2 the map $j_{n-1}: \mathbf{r a t}_{n-1}(2) \hookrightarrow \mathbf{r a t}_{n}(2)$ is a homology equivalence up to dimension $n-1$. This implies that $j_{n-1}$ is a homotopy equivalence up to dimension $k$ and, in particular, that the map $\pi_{k} \mathbf{r a t}_{n-1}(2) \hookrightarrow \pi_{k} \mathbf{r a t}_{n}(2)$ is surjective. But, as $j_{n-1}$ induces an isomorphism of fundamental groups, this means that the action of $\pi_{1} \boldsymbol{r a t}_{n}(2)$ on $\pi_{k} \boldsymbol{r a t}_{n}(2)$ is trivial, and, hence, $\boldsymbol{r a t}_{n}(2)$ is simple up to dimension $k+1$.

\section{The DEGREE OF AN ORNAMENT}

A 3-ornament is a smooth map of the disjoint union of 3 circles into $\mathbf{R}^{2}$, such that images of all three circles do not pass through the same point, see [13], [15]. Two 3-ornaments are equivalent if they are connected by a path in the space of such maps.

The most basic example of an (unoriented) ornament is shown on the right-hand side of Figure 1; we considered it as a generator of $\pi_{1} T(2,2)$. In fact, any 3-ornament defines an element of $\pi_{1} T(\mathrm{ev}, 2)$ in a similar fashion unless it has an infinite number of intersections with some horizontal line. However, this is a condition of infinite codimension, so we can always deform a 3 -ornament so that it does define an element of $\pi_{1} T(\mathrm{ev}, 2)=\mathbf{Z}$, which we call the degree of the ornament. Clearly, equivalent ornaments have the same degree.

The reason for calling this invariant "degree" is the following interpretation of it. Consider a regular ornament, i.e. such that it is an immersion and all the multiple 
points of its image in $\mathbf{R}^{2}$ are double transversal intersection points only. Let $f_{1}, f_{2}$ and $f_{3}$ be functions from $\mathbf{R}^{2}$ to $\mathbf{R}$, such that the zero set of each function defines the corresponding component of the ornament (up to parametrization, of course) and the zero set of each of the functions contains only a finite number of critical points. Assume also that at infinity $f_{i} \rightarrow 1, i=1,2,3$. Then we have a continuous map

$$
S^{2}=\mathbf{R}^{2} \cup\{\infty\} \stackrel{f_{1}, f_{2}, f_{3}}{\longrightarrow} \mathbf{R}^{3} \backslash 0 \simeq S^{2} .
$$

The degree of the ornament is just the degree of this map.

Even though the above construction cannot be found in [13], [15], historically it precedes not only ornaments, but, in fact, the very notion of degree. It can be found, in a more general setting, in Kronecker's paper [7], where he defines the "characteristic" of a system of functions with no common zeros. Kronecker's definition of the characteristic of the system $\left(f_{1}, f_{2}, f_{3}\right)$ was roughly as follows.

Suppose that the zero sets of functions $f_{i}$ intersect transversally. To each point of intersection $P_{k}$ of the curves $f_{1}=0$ and $f_{2}=0$ we assign an integer which is equal to 0 if $f_{3}\left(P_{k}\right)<0$, and \pm 1 if $f_{3}\left(P_{k}\right)>0$ and the tangent vectors to the curves $f_{1}=0$ and $f_{2}=0$ at $P_{k}$ define a positive/negative orientation of $\mathbf{R}^{2}$. (We assume that the orientations of the curves $f_{i}=0$ are induced by the standard orientation of $\mathbf{R}^{2}$.) The characteristic of the system of functions $\left(f_{1}, f_{2}, f_{3}\right)$ is the sum of these integers over all $P_{k}$.

It is clear that this definition amounts to calculating the algebraic number of inverse images of a point in $S^{2}$, so the Kronecker characteristic of a system $\left(f_{1}, f_{2}, f_{3}\right)$ is the same thing as the degree of a map given by these functions. Modern references for the Kronecker characteristic are [6] and, less recent, but probably more precise, 9 .

I would like to thank A.B. Merkov for his remark that there is a characteristictype formula for the degree of an ornament, and V.I. Arnold for pointing out that it was, in fact, first discovered by Kronecker.

\section{REFERENCES}

[1] C. Boyer, J. Hurtubise, B. M. Mann and R. J. Milgram, The topology of the space of rational maps into generalized flag manifolds. Acta Math. 173 (1994), 61-101. [MR 95h:55007

[2] C. Boyer, J. Hurtubise and R. J. Milgram, Stability theorems for spaces of rational curves. Preprint, 1999, math.AG/9903099

[3] R. W. Brockett, Some geometric questions in the theory of linear systems. IEEE Trans. Automat. Control 21 (1976), 449-455. MR 57:9177b

[4] M. A. Guest, The topology of the space of rational curves on a toric variety. Acta Math. 174 (1995), 119-145. MR 95k:58021

[5] M. A. Guest, A. Kozlowski and K. Yamaguchi, Spaces of polynomials with roots of bounded multiplicity. Fund. Math. 161 (1999), 93-117.

[6] M. Hirsch, Differential Topology. Springer-Verlag, 1976. MR 56:6669

[7] L. Kronecker, Über Systeme von Funktionen mehrerer Variabeln. Monatsberichte Berl. Acad. (1869), 159-193 and 688-698.

[8] A. Kozlowski and K. Yamaguchi, Topology of complements of discriminants and resultants. J. Math. Soc. Japan 52 (2000), 949-959. CMP 2000:16

[9] S. Lefschetz, Topology. AMS Colloquium Publications, New York, 1930.

[10] D. McDuff, Configuration spaces of positive and negative particles. Topology, 14 (1975), 91-107. MR 50:11225

[11] J. Milnor, Morse Theory, revised edition. Princeton, 1969. MR 29:634

[12] G. Segal, The topology of spaces of rational functions. Acta Math. 143 (1979), 39-72. MR 81c:55013 
[13] V. A. Vassiliev, Invariants of ornaments in: Singularities and bifurcations. Adv. Soviet Math. vol. 21, Amer. Math. Soc., Providence, RI, 1994, pp. 225-262. [MR 96a:57029]

[14] V. A. Vassiliev, Topology of discriminants and their complements. Proceedings of the International Congress of Mathematicians, Vol. 1, 2 (Zürich, 1994) 209-226, Birkhäuser, Basel, 1995. MR 97j:57041

[15] V. A. Vassiliev, Complements of discriminants of smooth maps: topology and applications, revised edition. Translations of Math. Monographs 98. Amer. Math. Soc., Providence, RI, 1994. MR 94i:57020

[16] K. Yamaguchi, Complements of resultants and homotopy types, J. Math. Kyoto Univ. 39 (1999), 675-684. CMP 2000:08

Instituto de Matemáticas (Unidad Cuernavaca), Universidad Nacional Autónoma de MÉxico, A.P. 273-3, C.P. 62251, Cuernavaca, Morelos, México

E-mail address: jacob@matcuer.unam.mx 